\title{
Magnetically Guided Fast Electrons in Cylindrically Compressed Matter
}

F. Pérez, ${ }^{1, *}$ A. Debayle, ${ }^{2}$ J. Honrubia, ${ }^{2}$ M. Koenig, ${ }^{1}$ D. Batani, ${ }^{3}$ S. D. Baton, ${ }^{1}$ F. N. Beg, ${ }^{4}$ C. Benedetti, ${ }^{5}$ E. Brambrink,${ }^{1}$ S. Chawla, ${ }^{4,6}$ F. Dorchies, ${ }^{7}$ C. Fourment, ${ }^{7}$ M. Galimberti, ${ }^{8}$ L. A. Gizzi, ${ }^{9}$ L. Gremillet, ${ }^{10}$ R. Heathcote, ${ }^{8}$ D. P. Higginson, ${ }^{4,6}$ S. Hulin, ${ }^{7}$ R. Jafer, ${ }^{3}$ P. Koester, ${ }^{9}$ L. Labate, ${ }^{9}$ K. L. Lancaster, ${ }^{8}$ A. J. MacKinnon, ${ }^{6}$ A. G. MacPhee, ${ }^{6}$ W. Nazarov, ${ }^{11}$ P. Nicolai, ${ }^{7}$ J. Pasley, ${ }^{12,8}$ R. Ramis, ${ }^{2}$ M. Richetta, ${ }^{13}$ J. J. Santos, ${ }^{7}$ A. Sgattoni,${ }^{5}$ C. Spindloe, ${ }^{8}$ B. Vauzour, ${ }^{7}$ T. Vinci, ${ }^{1}$ and L. Volpe ${ }^{3}$

${ }^{1}$ LULI, École Polytechnique, CNRS, CEA, UPMC, 91128 Palaiseau Cedex, France

${ }^{2}$ ETSI Aeronáuticos, Universidad Politécnica de Madrid, Spain

${ }^{3}$ Dipartimento di Fisica, Università di Milano-Bicocca, Italy

${ }^{4}$ University of California, San Diego, La Jolla, California 92093, USA

${ }^{5}$ Dipartimento di Fisica, Università di Bologna, Bologna, Italy

${ }^{6}$ Lawrence Livermore National Laboratory, Livermore, California 94550, USA

${ }^{7}$ Université de Bordeaux-CNRS-CEA, Centre Lasers Intenses et Applications (CELIA), Talence, F-33405, France

${ }^{8}$ Central Laser Facility, Rutherford Appleton Laboratory, Didcot, United Kingdom

${ }^{9}$ ILIL, Istituto Nazionale di Ottica, UOS Adriano Gozzini, CNR, Via G. Moruzzi 1, Pisa, Italy

${ }^{10}$ CEA, DAM, DIF, F-91297 Arpajon, France

${ }^{11}$ University of St. Andrews, Fife KY16 9AJ, Scotland, United Kingdom

${ }^{12}$ York Plasma Institute, Department of Physics, University of York, York, YO10 5DQ, United Kingdom

${ }^{13}$ Dipartimento di Ingegneria Meccanica, Università di Roma Tor Vergata, Italy

(Received 1 February 2011; published 4 August 2011)

Fast electrons produced by a 10 ps, $160 \mathrm{~J}$ laser pulse through laser-compressed plastic cylinders are studied experimentally and numerically in the context of fast ignition. $K_{\alpha}$-emission images reveal a collimated or scattered electron beam depending on the initial density and the compression timing. A numerical transport model shows that implosion-driven electrical resistivity gradients induce strong magnetic fields able to guide the electrons. The good agreement with measured beam sizes provides the first experimental evidence for fast-electron magnetic collimation in laser-compressed matter.

Understanding the transport of relativistic electron beams (REB) through an inhomogeneous compressed plasma is a prerequisite for the success of the fast ignition (FI) scheme for inertial confinement fusion [1]. In this design, an imploded fuel capsule is heated by a REB generated by a relativistic-intensity, picosecond laser pulse at maximum compression. A major challenge is to ensure good collimation of these electrons so as to heat the dense core up to ignition temperatures [2].

Recent studies revealed that REBs originating from the interaction of an intense laser pulse with a solid-density target may be strongly divergent [3]. State-of-the-art integrated simulations indicate that self-generated magnetic fields [4] could be unable to compensate for this large angular spread, which may lead to a poor electron-tocore coupling efficiency [2]. Besides, it has been found that cone-guided targets, designed to bring the electron source closer to the compressed core, may induce additional electron losses in case of a low laser contrast [5]. These features may not apply to a full-scale FI scheme, and this concern motivated the experiment presented in this Letter. Indeed, laser-compressed targets were employed instead of the usual cold solid targets, in order to ensure conditions closer to FI. As shown further below, this brings forward an efficient electron collimation mechanism [4]: magnetic fields generated by the REB current in the presence of strong gradients of the electrical resistivity (henceforth denoted by $\eta$ ) tend to deflect the electrons towards high- $\boldsymbol{\eta}$ regions. This effect, recently evidenced inside structured solid targets made of materials of varying initial resistivity, either transversally [6] or longitudinally [7], could serve to collimate the REB through a high- $\eta$ channel pointed towards the compressed core. In our case of lasercompressed targets, one could consider taking advantage of the $\eta$ gradients caused by the density and temperature inhomogeneities spontaneously arising during the fuel compression. If arranged properly, the resulting fields could guide the REB. In this Letter, we present the first experimental evidence for such magnetic collimation inside laser-compressed matter. Our major finding, supported by numerical simulations, is that the efficiency of this mechanism critically depends on the transient resistivity profile experienced by the REB during the compression.

The experiment, detailed in Ref. [8], was conceived within the HiPER project experimental road map [9], and carried out on the VULCAN TAW laser facility of the Rutherford Appleton Laboratory. As illustrated in Fig. 1, cylindrical targets were compressed by four long-pulse (LP) beams individually synchronized with a precision better than $100 \mathrm{ps}$. Each delivered 40 to $70 \mathrm{~J}$ at a 


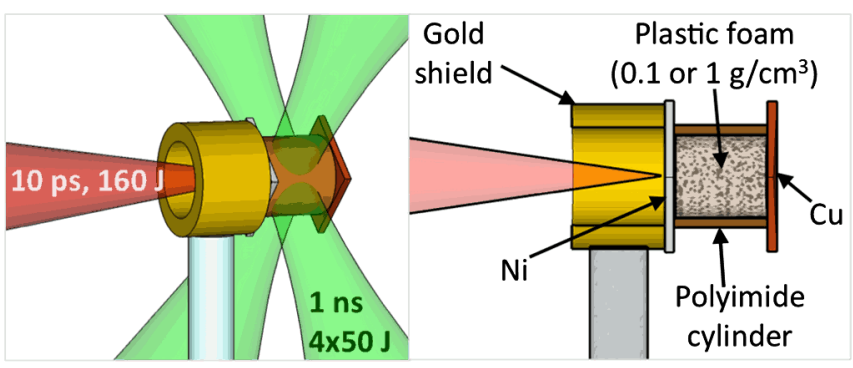

FIG. 1 (color online). Schematics of the laser beam geometry and target setup.

wavelength of $0.53 \mu \mathrm{m}$ within a $150 \mu \mathrm{m}$ (FWHM) focal spot during 1 ns with a $\sim 100$ ps rising time. Fast electrons were then generated by a 160-J, 10-ps short-pulse (SP) laser beam. Using a $f / 3$ off-axis parabola, it was focused to a $20 \mu \mathrm{m} \mathrm{FWHM} \mathrm{spot,} \mathrm{thus} \mathrm{yielding} \mathrm{an} \mathrm{average} \mathrm{intensity}$ of $5 \times 10^{18} \mathrm{~W} / \mathrm{cm}^{2}$ at a wavelength of $1.053 \mu \mathrm{m}$. The delay $\tau$ between the LPs and the SP was varied from 0 to $3 \mathrm{~ns}$ in order to inject the REB at different stages of the compression. The time origin $\tau=0 \mathrm{~ns}$ corresponds to the SP hitting the target at the beginning of the LPs. Note that a \pm 100 ps jitter in the SP synchronization results in the error bars displayed in Fig. 2.

As detailed in Fig. 1, the targets were made of a polyimide tube filled with plastic foam (TMPTA) available in two different densities (either 0.1 or $1 \mathrm{~g} / \mathrm{cm}^{3}$ ). The tube was $200 \mu \mathrm{m}$ long, $220 \mu \mathrm{m}$ wide, and had a $20 \mu \mathrm{m}$-thick wall. It was closed on both sides by $20 \mu \mathrm{m}$ metal foils of either $\mathrm{Ni}$ or $\mathrm{Cu}$. Fast electrons were accelerated on the $\mathrm{Ni}$ side towards the $\mathrm{Cu}$ side. An empty plastic-coated Au tube was used as a shield against the ablated plasma.

The target compression, predicted by the hydrodynamic code CHIC [10], was experimentally verified using proton and $\mathrm{x}$-ray radiography [11]. The electron transport measurements have been reported elsewhere [8] and are briefly summarized here. The REB size was measured at the rear surface of the plastic cylinder, i.e., the $\mathrm{Cu}$ layer, using a standard $K_{\alpha}$ x-ray imaging technique [12]. Coupled with an imaging plate detector, a spherically bent quartz crystal

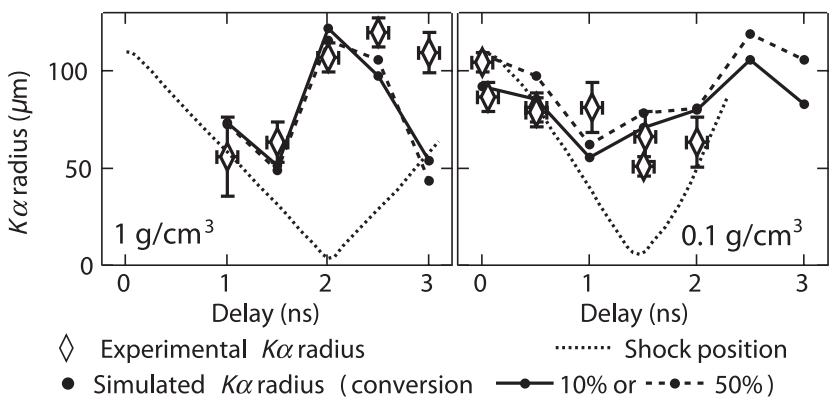

FIG. 2. Experimental and hybrid simulations $K_{\alpha}$ signal radius as a function of the delay $\tau$, for 1 (left) or $0.1 \mathrm{~g} / \mathrm{cm}^{3}$ (right) foams. The dotted line represents the shock front position simulated by MULTI3D. with a $1.541 \AA$ interatomic distance and a radius of curvature of $380 \mathrm{~mm}$ produced side-view $\mathrm{x}$-ray images around the $\mathrm{Cu}-K_{\alpha 1}$ line (at $8052 \pm 5 \mathrm{eV}$ ). Its magnification was $\sim 10$ and its spatial resolution between 10 and $20 \mu \mathrm{m}$. The obtained time-integrated images show the regions of the $\mathrm{Cu}$ layer crossed by fast electrons (with energies $>8 \mathrm{keV}$ ). The size of the x-ray image is used to estimate the REB transverse size across the rear surface, and therefore to assess its collimation or divergence. Figure 2 plots this $K_{\alpha}$ signal radius as a function of the delay (or injection time) $\tau$. Two distinct behaviors are found depending on the initial foam density. High-density foams $\left(1 \mathrm{~g} / \mathrm{cm}^{3}\right)$ lead to a broadened signal when the compression is important, whereas the trend is reversed for low-density foams $\left(0.1 \mathrm{~g} / \mathrm{cm}^{3}\right)$. A striking feature of the $1 \mathrm{~g} / \mathrm{cm}^{3}$ targets is the "jump" observed between $\tau=1.5$ and 2 ns. Threedimensional hydrodynamic simulations by the code MULTI3D [13] reveal that this sudden variation coincides with the convergence of the compression shock wave on the cylinder axis (see Fig. 2): for a reason that will be clarified below, the REB happens to be more strongly scattered when injected after the shock convergence. At $0.1 \mathrm{~g} / \mathrm{cm}^{3}$, by contrast, the measured size weakly decreases if the injection occurs after the shock collapse (after $\tau=1.5 \mathrm{~ns}$ ).

These results may be attributed to several mechanisms, such as collisional slowing-down or scattering, or perturbation of the front Ni layer which can alter the fast-electron source (this layer being mostly affected at late times, it would be unlikely to modify the general behavior). The electron trajectories may also be influenced by the selfconsistent resistive fields, highly sensitive to the temperature and density gradients. To understand the REB transport through the compressed foam, we performed 2D axisymmetric hybrid simulations [14] using the density and temperature profiles predicted by MULTI3D (examples of these initial profiles are shown in the top panels of Figs. 3-5). As these profiles are not axisymmetric [8],

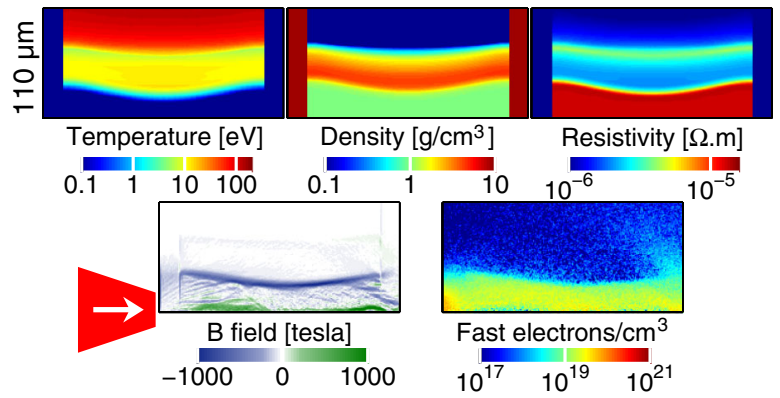

FIG. 3 (color online). Input temperature, density, and resistivity for the hybrid code, with a $1 \mathrm{~g} / \mathrm{cm}^{3}$ foam, at $\tau=1.5 \mathrm{~ns}$ (the shock wave has not yet collapsed). The simulated magnetic field and fast-electron density are plotted after 15 ps of transport, showing a collimated REB. In all cases, half of the cylinder is represented. 


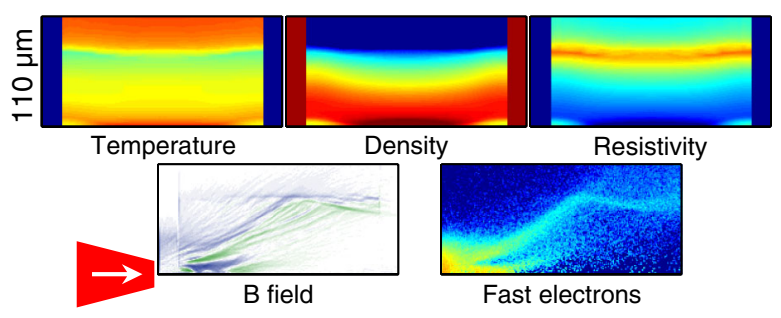

FIG. 4 (color online). Same plots and same color scales as in Fig. 3, also with a $1 \mathrm{~g} / \mathrm{cm}^{3}$ foam, but at $\tau=2 \mathrm{~ns}$ (the shock wave has collapsed so the REB is not collimated).

they were azimuthally averaged. The azimuthal nonuniformities of the compressed target are assumed to have little effect on the overall electron transport and are neglected in our model.

The input REB energy and angular distributions to the hybrid code were estimated from a $2 \mathrm{D}$ collisionless particle-in-cell simulation using the code CALDER [15]. Because of computing limitations, the laser pulse was reduced to a 0.5 ps linear ramp followed by a constant intensity of $5 \times 10^{18} \mathrm{~W} / \mathrm{cm}^{2}$, during an additional $1 \mathrm{ps}$. The density profile along the laser direction was taken from a DUED [16] 2D hydrodynamic simulation of the preplasma, generated by a $500 \mathrm{ps}, 10^{13} \mathrm{~W} / \mathrm{cm}^{2}$ pedestal focused on the front $\mathrm{Ni}$ layer. It predicted a $\sim 4 \mu \mathrm{m}$ separation between the critical density $\left(n_{c}\right)$ region and the bulk target $\left(50 n_{c}\right)$. The REB data were extracted $\sim 15 \mu \mathrm{m}$ behind the laser-plasma interaction region. Its angular distribution was fitted to the model function $f(\theta)=\exp \left[-\left(\theta-\theta_{r} \arctan \left(r / r_{0}\right)\right)^{2} / \Delta \theta_{0}^{2}\right]$ with the dispersion angle $\Delta \theta_{0}=30^{\circ}$ and the mean radial angle $\theta_{r}=30^{\circ}$ [17]. The low-energy component $(0.2$ to $3 \mathrm{MeV}$ ) of the electron energy distribution was approximated to a decreasing exponential with a mean energy $\sim 600 \mathrm{keV}$, comparable to the ponderomotive scaling [18].

The REB parameters described above were assumed as initial conditions in the hybrid code, with the exception of the laser-to-fast-electron conversion efficiency that was considered as a free parameter. The REB transport was simulated during $30 \mathrm{ps}$ through the Ni foil, the compressed cylinder, and the $\mathrm{Cu}$ foil. Specular reflection was applied at the rear boundary to mimic the self-induced electrostatic

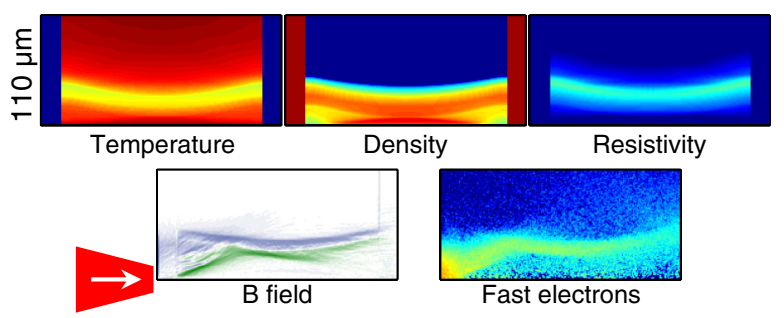

FIG. 5 (color online). Same as Fig. 4, but with a $0.1 \mathrm{~g} / \mathrm{cm}^{3}$ foam at $\tau=1.5 \mathrm{~ns}$. The coronal plasma allows for a collimated beam even though the shock wave has collapsed. field at the target rear surface. The plastic foam electrical resistivity was computed using the classical Drude model $\eta=m \nu / e^{2} n_{e}$, where $n_{e}$ is the background electron density, and $\nu$ the electron-ion collision frequency, expressed as a harmonic mean $\nu^{-1}=\nu_{\mathrm{sp}}^{-1}+\nu_{\mathrm{sat}}^{-1}$ between the maximum collision frequency $\nu_{\text {sat }}=\sqrt{k_{B} T_{e} / m_{e}}\left(4 \pi n_{i} / 3\right)^{1 / 3}$ and the Spitzer collision frequency $\nu_{\mathrm{sp}}\left(n_{i}\right.$ being the ion density and $T_{e}$ the electron temperature). Other details on the hybrid code, as the ionization model, are described in Ref. [14]. $K_{\alpha}$ emission, calculated with the model of Ref. [19], was analyzed in the same manner as the experimental data.

The simulated $K_{\alpha}$ spot size variation vs injection time is compared to the experimental results in Fig. 2 for two values of the conversion efficiency (10\% and 50\%). The experimental trends are well reproduced, particularly the jump between $\tau=1.5$ and $2 \mathrm{~ns}$ in the $1 \mathrm{~g} / \mathrm{cm}^{3}$ case. This good general agreement proves that the hybrid model accurately accounts for the main processes of the electron transport, among which the self-consistent magnetic field generation through resistivity gradients appears dominant. Note that the discrepancy for $\tau=3 \mathrm{~ns}\left(1 \mathrm{~g} / \mathrm{cm}^{3}\right.$ targets $)$ is due to the front surface deformation after the shock collapse, which changes the electron divergence.

Assuming cylindrical geometry and almost perfect neutralization of the REB current $\mathbf{j}_{b}$ by a strongly collisional return current, an azimuthal resistive magnetic field is induced according to the following combination of Ohm's and Faraday's laws: $\partial_{t} B_{\theta}=\partial_{z}\left(\eta j_{b r}\right)-\partial_{r}\left(\eta j_{b z}\right)$. Assuming $\partial_{z}\left(\eta j_{b r}\right) \ll \partial_{r}\left(\eta j_{b z}\right)$ for a forward-directed REB and $\eta \partial_{r} j_{b z} \ll j_{b z} \partial_{r} \eta$ for a smooth transverse current profile, one obtains $\partial_{t} B_{\theta} \simeq-j_{b z} \partial_{r} \eta$. The resulting field thus deflects the fast electrons towards high- $\eta$ regions. In the case of a preionized plastic target, resistivity gradients mostly develop in two distinct regimes depending on the electron temperature. For temperatures around the Fermi temperature, $T_{e} \sim T_{F}$, the resistivity gradient is mainly due to the density gradient, $\partial_{r} \eta \sim \partial_{r} n_{e}^{-2 / 3}$, and the resistive magnetic field bends the electrons' trajectories towards low-density regions. When $T_{e} \gg T_{F}$, the resistivity gradient depends only on the temperature: $\partial_{r} \eta \sim \partial_{r} T_{e}^{-3 / 2}$. In this Spitzer regime, electron trajectories are bent towards low-temperature regions. During the compression, both types of $\eta$ gradients may arise and magnetically shape the REB according to their structure.

To illustrate this, let us investigate the jump in the $K_{\alpha}$ spot size observed between $\tau=1.5$ and $2 \mathrm{~ns}$ for the $1 \mathrm{~g} / \mathrm{cm}^{3}$ foam. Let us first consider the case $\tau=1.5 \mathrm{~ns}$. The corresponding plasma temperature, density, and resistivity are shown in the top panels of Fig. 3. At that stage, the shock wave driven by the LPs has not yet collapsed onto the cylinder axis: the on-axis density minimum yields a negative $\eta$ gradient located at $r \sim 30 \mu \mathrm{m}$ away from the axis, i.e., close to the REB source. There follows a magnetic field strong enough to efficiently collimate the REB 
within the central low-density region, as displayed in the bottom panels of Fig. 3. Indeed, more than $60 \%$ of the electrons stay in this $30 \mu \mathrm{m}$-radius channel. By $\tau=2 \mathrm{~ns}$, the shock has reached the axis and the peak $\eta$ gradient is induced by $T_{e}$ variations in the tenuous, hot coronal plasma (at $r \sim 70 \mu \mathrm{m}$, see Fig. 4). Being much weakened and driven further away from the axis, the resulting field is no longer able to confine the electrons within a narrow channel. In summary, the sudden increase in the $K_{\alpha}$ radius found in the $1 \mathrm{~g} / \mathrm{cm}^{3}$ case stems from the disappearance of the shock front-driven magnetic field at the time of the shock collapse.

No such jump is observed in the case of the lightest foam $\left(0.1 \mathrm{~g} / \mathrm{cm}^{3}\right)$. As shown in Fig. 5, after the shock collapse $(\tau=1.5 \mathrm{~ns})$, the whole plasma is in the Spitzer regime, yet the hot coronal plasma is located closer to the axis $(r \sim 30 \mu \mathrm{m})$ than for the dense foam. The temperaturedriven resistivity gradient arising at the ablation surface therefore leads to an efficient collimating field, which substitutes to the shock-driven guiding field developing prior to the shock collapse.

The overall behavior of the REB has been understood and shown to be dominated by resistive magnetic fields. Additional simulations that have been carried out with varying conversion efficiency (see Fig. 2), electron mean energy, divergence, and resistivity saturation value, show that the collimation mechanism is not highly sensitive to these input parameters, and consequently support the robustness of our scenario. Neglecting collisional effects in the CALDER simulations seems therefore justified. Note, however, that the resistive filamentation instability [20] is modeled by the hybrid simulations, yielding transverse modulations in Figs. 3-5.

Let us emphasize that the mechanism presented here differs from the one simulated by Nakamura et al. [21] in the case of a cylindrical target compressed to a very narrow radius $(\sim 2 \mu \mathrm{m})$, wherein collimation ensues from electrostatic transverse confinement. This process, similar to that occurring through a solid wire, requires $n_{e}$ lower than the REB density beyond the compression radius, which never happens in our case. Instead, collimation here proceeds from the resistive magnetic field induced either by the density gradient at the shock front or the temperature gradient at the ablation surface, if close enough to the REB radius.

In conclusion, the transport of an ultrahigh current laserproduced electron beam through compressed plastic cylinders has been spatially characterized by means of $\mathrm{Cu}-K_{\alpha}$ imaging. The measured and simulated evolution of the $K_{\alpha}$ spot size vs the injection time revealed that the electrons can be collimated by resistive magnetic fields developing either at the shock front or in the coronal plasma. The collimation induced at the shock front appears to be the most efficient, and can be controlled by adjusting the fastelectron synchronization with the compression pulses. This constitutes the first experimental evidence for fast-electron collimation in laser-compressed matter. In the fast ignition scheme, similar collimating fields from transverse resistivity gradients may arise from inhomogeneities induced by the inserted cone or by the hole-boring mechanism.

The authors acknowledge the support of the HiPER project and Preparatory Phase Funding Agencies (EC, MSMT and STFC) in undertaking this work. The numerical study was supported by Grant No. ENE2009-11668 of the Spanish Ministry of Education and Research and by the European SILMI program. The authors thankfully acknowledge the computer resources, technical expertise, and assistance provided by the CeSViMa. This work was performed using HPC resources from GENCI-[CCRT/ CINES] (Grant No. 2010-x2010056304).

*frederic.perez@polytechnique.edu

[1] M. Tabak et al., Phys. Plasmas 1, 1626 (1994).

[2] J. J. Honrubia and J. Meyer-ter-Vehn, Plasma Phys. Controlled Fusion 51, 014008 (2009).

[3] J. S. Green et al., Phys. Rev. Lett. 100, 015003 (2008); F. Perez et al., Phys. Plasmas 17, 113106 (2010).

[4] A. R. Bell and R. J. Kingham, Phys. Rev. Lett. 91, 035003 (2003).

[5] S. D. Baton et al., Phys. Plasmas 15, 042706 (2008); A. G. MacPhee et al., Phys. Rev. Lett. 104, 055002 (2010).

[6] S. Kar et al., Phys. Rev. Lett. 102, 055001 (2009); B. Ramakrishna et al., Phys. Rev. Lett. 105, 135001 (2010).

[7] L. A. Gizzi et al., Phys. Rev. ST Accel. Beams 14, 011301 (2011).

[8] F. Perez et al., Plasma Phys. Controlled Fusion 51, 124035 (2009).

[9] D. Batani et al., AIP Conf. Proc. 1209, 129 (2010).

[10] P. Maire et al., SIAM J. Sci. Comput. 29, 1781 (2007).

[11] L. Volpe et al., Phys. Plasmas 18, 012704 (2011); B. Vauzour et al., Phys. Plasmas 18, 043108 (2011).

[12] R. B. Stephens et al., Phys. Rev. E 69, 066414 (2004).

[13] R. Ramis, J. Meyer-ter-Vehn, and J. Ramírez, Comput. Phys. Commun. 180, 977 (2009).

[14] J. J. Honrubia et al., Phys. Plasmas 12, 052708 (2005).

[15] E. Lefebvre et al., Nucl. Fusion 43, 629 (2003).

[16] S. Atzeni et al., Comput. Phys. Commun. 169, 153 (2005).

[17] A. Debayle et al., Phys. Rev. E 82, 036405 (2010).

[18] S. C. Wilks et al., Phys. Rev. Lett. 69, 1383 (1992).

[19] C. Hombourger, J. Phys. B 31, 3693 (1998).

[20] L. Gremillet, G. Bonnaud, and F. Amiranoff, Phys. Plasmas 9, 941 (2002); A. Karmakar et al., Phys. Rev. Lett. 101, 255001 (2008).

[21] H. Nakamura et al., Phys. Rev. Lett. 100, 165001 (2008). 